International Journal of Modern Physics C

(2020) 2050099 (12 pages)

(C) World Scientific Publishing Company

DOI: $10.1142 /$ S0129183120500990

\title{
On the ideal weights for WENO/WENO-like finite difference schemes for the first derivative, I
}

\author{
Jian Kang* and Xinliang $\mathrm{Li}^{\dagger}$ \\ University of Chinese Academy of Sciences \\ No. 19 Yuquan Road, Shijingshan District \\ Beijing 100049, P. R. China \\ Institute of Mechanics, Chinese Academy of Sciences \\ No. 15 Beisihuanxi Road, Haidian District \\ Beijing 100190, P. R. China \\ *kangjian13@mails.ucas.ac.cn \\ †lixl@imech.ac.cn
}

Received 6 February 2020

Accepted 22 March 2020

Published 25 June 2020

\begin{abstract}
An interesting fact which was used in the construction of targeted essentially non-oscillatory (TENO) schemes is that a $(n+1)$ th order accurate scheme can be written as a linear combination of two $n$th order accurate schemes. Taking advantage of this fact, we propose a formula to determine the ideal weights used in developing finite difference WENO/WENO-like schemes. Such quantitative results are helpful for further discussion on finite difference WENO/WENOlike schemes.
\end{abstract}

Keywords: Finite difference; weighted essentially non-oscillatory; ideal weights; proof.

PACS Nos.: 47.11.+j, 47.11.Bc.

\section{Introduction}

The weighted essentially non-oscillatory (WENO) schemes ${ }^{1,2}$ are a class of shock capturing schemes that have been quite popular for the past two decades. When shock occurs, the WENO schemes assign small weights (approximately zero) to stencils containing jumps. This method is somewhat similar to ENO schemes, ${ }^{3-5}$ which only select the "smoothest" sub-stencil to perform further computations. When shock is not present, a higher order accurate flux will be computed using all the fluxes computed in the ENO schemes. Such a procedure improves the scheme's accuracy in smooth zones.

\footnotetext{
$\dagger$ Corresponding author.
} 
As far as we know, there are at least two kinds of methods to determine the ideal weights for WENO schemes. One kind of the methods is to determine the ideal weights by solving a system of linear equations. ${ }^{6}$ For example, with given point values $u_{i}(1 \leq i \leq 5)$ for the fifth-order WENO-JS scheme, we can first determine the numerical fluxes $f_{i}\left(u_{i}, u_{i+1}, u_{i+2}\right)(1 \leq i \leq 3)$ on all the three sub-stencils, and then determine the numerical flux $f\left(u_{1}, \ldots, u_{5}\right)$ on the whole stencil. The system of linear equations for the ideal weights $c_{i}(1 \leq i \leq 3)$ just reflects the fact that

$$
c_{1} f_{1}+c_{2} f_{2}+c_{3} f_{3}=f
$$

and the weights $c_{i}(1 \leq i \leq 3)$ are independent of $u_{i}(1 \leq i \leq 5)$. This example is for conservative schemes. The basic idea can also be used in the case of nonconservative schemes.

Another kind of methods of determining the ideal weights of WENO approximations employs recurrence relations. There exist recurrence relations for interpolation, integration, and reconstruction-approximation to derivatives. ${ }^{7}$ For the case of finite difference schemes, two kinds of recurrence relations are involved: one for conservative schemes, and the other for nonconservative schemes. The recently developed targeted ENO (TENO) schemes ${ }^{8-10}$ used the "recurrence relation" idea. When it comes to constructing high order accurate schemes, the TENO schemes take advantage of the fact that two $n$th order accurate schemes can construct a $(n+1)$ th order accurate scheme, by expressing the latter as a linear combination of the former.

The aforementioned fact is actually a recurrence relation. When we consider conservative schemes, this fact corresponds to Aitken-Neville interpolation. ${ }^{7}$ When we consider nonconservative schemes, this fact leads to computations of linear recurrence sequences. Thus the fact suggests a means of determining the ideal weights of finite difference WENO/WENO-like schemes. Regardless of conservative or nonconservative, the ideal weights are the same in both cases for uniform meshes since both cases lead to the same recurrence relation.

In this paper, we propose and prove an explicit formula for the ideal weights for finite difference WENO schemes on uniform meshes. The rest of this paper is organized as follows. In Sec. 2 a formula is introduced, which serves to determine the ideal weights for finite difference WENO schemes on uniform meshes. In Sec. 3, the formula is proved. Discussions on the ideal weights of upwind WENO schemes and those of TENO schemes are presented in Sec. 4. In Sec. 5, the conclusion is outlined.

\section{Main Result}

In order to introduce the main result, we introduce some definitions first.

Let $T_{h}$ be the shift operator with step $h$, which maps $f(x)$ to $f(x+h)$ :

$$
T_{h}[f](x)=f(x+h) .
$$

And $D$ stands for the differential operator, i.e.

$$
D=\frac{\mathrm{d}}{\mathrm{d} x} .
$$


If the function $f(x+i y)$, considered as a function of complex variable $x+i y$, is entire (i.e. $f(x+i y)$ is analytic at all finite points of the complex plane $\mathbb{C}$ ), then the Taylor series

$$
f(x+h)=f(x)+\sum_{i=1}^{\infty} \frac{h^{i}}{i !} f^{(i)}(x)
$$

converge for all real number $x$, and the convergence radius is $\infty$. Thus, we have in that case

$$
T_{h}=I+\frac{h}{1 !} D+\frac{h^{2}}{2 !} D^{2}+\cdots=e^{h D}, \quad \forall h \in \mathbb{R},
$$

where $I$ represents the identity operator which maps $f(x)$ to itself:

$$
I[f](x)=f(x) .
$$

The rest of our discussion is based on (5). With $T_{h}$ and $D$, we define the highest order accurate finite difference schemes as follows.

Definition 1. Suppose $p, q \in \mathbb{Z}$ satisfy $p<q$, and $h \in \mathbb{R}^{+}$. The operator $\tilde{D}_{h, p, q}$ is defined as

$$
\tilde{D}_{h, p, q}=\frac{1}{h} \sum_{i=p}^{q} a_{i} T_{i h}
$$

where $a_{i}(p \leq i \leq q)$ satisfy

$$
\sum_{i=p}^{q} a_{i} T_{i h}=\frac{h}{1 !} D+\sum_{n=q-p+1}^{\infty} \frac{h^{n}}{n !} b_{n} D^{n} .
$$

Remark 1. In Definition 1, a uniform mesh is assumed, i.e. the points $x_{i}=i h$ (where $i \in \mathbb{Z}$ ) are evenly distributed on the real axis. The scheme (operator) $\tilde{D}_{h, p, q}$ uses consecutive grid points: $p$ and $q$ in the subscript indicate where to start and where to end, respectively. Note that $p$ and $q$ are indices of the grid points.

Using the knowledge on the Vandermonde determinant, one can easily verify that the weights $a_{i}$ occurring in Definition 1 are uniquely determined.

Now, we are ready to give the main theorem.

Theorem 1. Suppose $p, q, n \in \mathbb{Z}$ satisfy $p+n \leq 0, p+q \geq 0, n \geq 0, q \geq 1$. Then

$$
\tilde{D}_{h, p, p+q+n}=\sum_{i=0}^{n} \frac{\left(\begin{array}{c}
-p \\
i
\end{array}\right)\left(\begin{array}{c}
p+q+n \\
n-i
\end{array}\right)}{\left(\begin{array}{c}
q+n \\
n
\end{array}\right)} \tilde{D}_{h, p+i, p+q+i}
$$

Remark 2. In Theorem 1, $p$ represents the leftmost index of the whole stencil, while $q$ equals to the rightmost index minus the leftmost index for all sub-stencils. The 
integer $n$ represents the difference between the leftmost index of the rightmost substencil and that of the leftmost sub-stencil.

The inequalities $p+n \leq 0$ and $p+q \geq 0$ actually mean that all the sub-stencils contain the same grid point, i.e. the point at which the derivative is evaluated. The inequality $n \geq 0$ means that the total of sub-stencils is no less than 1 . The inequality $q \geq 1$ means that each sub-stencils should contain at least two grid points (see Fig. 1).

The ideal weights turn out to be rational functions of binomial coefficients. Generally, given nonnegative integers $a, b$ such that $a \geq b$, the binomial coefficient $\left(\begin{array}{l}a \\ b\end{array}\right)$ can be computed via the following formula:

$$
\left(\begin{array}{l}
a \\
b
\end{array}\right)=\frac{a !}{(a-b) ! b !},
$$

where $a$ ! represents factorial:

$$
a !=\prod_{i=1}^{a} i=1 \times 2 \times \cdots \times a \quad(a \geq 1)
$$

and $0 !=1$. The formulae of $(a-b)$ ! and $b$ ! can be deduced by analogy.

\section{Proof of the Main Theorem}

In this section, we prove Theorem 1. Before giving the proof of the main theorem, we would like to introduce and prove three lemmas.

Lemma 1. Suppose $x_{1}, \ldots, x_{n} \in \mathbb{R}$, and

$$
s_{k}=\sum_{i=1}^{n} a_{i} x_{i}^{k}, \quad k \geq 0
$$

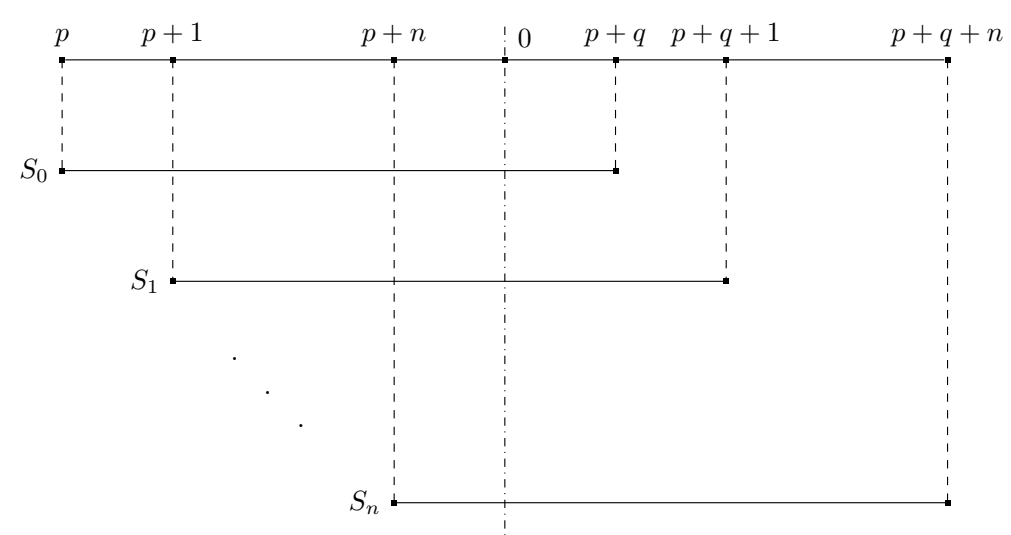

Fig. 1. A line plot to aid understanding the meanings of parameters $p, q$ and $n$. The sub-stencils are $S_{0}$, $S_{1}, \ldots, S_{n}$. Note that all the sub-stencils contain the index 0 . 
$c_{0}, c_{1}, \ldots, c_{n} \in \mathbb{R}$ satisfy

$$
\sum_{i=0}^{n} c_{i} x^{i}=\prod_{j=1}^{n}\left(x-x_{j}\right)
$$

Then $\forall k \in \mathbb{Z}^{+} \cup\{0\}$,

$$
\sum_{i=0}^{n} c_{i} s_{k+i}=0
$$

Here, we define $0^{0}=1$.

Proof. From (13), we have

$$
\sum_{j=0}^{n} c_{j} x_{i}^{j}=0, \quad 1 \leq i \leq n
$$

Multiplying $a_{i} x_{i}^{k}$ on both sides of (15) gives

$$
\sum_{j=0}^{n} a_{i} c_{j} x_{i}^{k+j}=0, \quad 1 \leq i \leq n .
$$

Thus

$$
0=\sum_{i=1}^{n} \sum_{j=0}^{n} a_{i} c_{j} x_{i}^{k+j}=\sum_{j=0}^{n} c_{j} \sum_{i=1}^{n} a_{i} x_{i}^{k+j}=\sum_{j=0}^{n} c_{j} s_{k+j} .
$$

Lemma 2. Suppose $p, q \in \mathbb{Z}$ satisfy $p<q$, and

$$
\tilde{D}_{h, p, q}=D+\sum_{i=q-p+1}^{\infty} \frac{h^{i-1}}{i !} b_{i} D^{i}
$$

Then

$$
b_{q-p+1}=(-1)^{q-p+1} \sum_{i=p}^{q} \prod_{\substack{p \leq j \leq q \\ j \neq i}} j .
$$

Proof. By Definition $1, \exists a_{p}, \ldots, a_{q}$ such that

$$
\sum_{i=p}^{q} a_{i} T_{i h}=\frac{h}{1 !} D+\sum_{i=q-p+1}^{\infty} \frac{h^{i}}{i !} b_{i} D^{i}
$$

If we rewrite (20) as

$$
\sum_{i=p}^{q} a_{i} T_{i h}=\sum_{i=0}^{\infty} \frac{h^{i}}{i !} b_{i} D^{i} .
$$


Then

$$
b_{k}=\sum_{i=p}^{q} a_{i} i^{k}, \quad \forall k \in \mathbb{Z}^{+} \cup\{0\} .
$$

Let

$$
\prod_{i=p}^{q}(x-i)=\sum_{i=0}^{q-p+1} c_{i} x^{i}
$$

By Lemma 1,

$$
\sum_{i=0}^{q-p+1} c_{i} b_{i}=0
$$

It is clear that

$$
b_{0}=0, \quad b_{1}=1, \quad b_{2}=\cdots=b_{q-p}=0 .
$$

So, we have

$$
c_{1} b_{1}+c_{q-p+1} b_{q-p+1}=0 .
$$

Note that $b_{1}=c_{q-p+1}=1$. (26) implies

$$
b_{q-p+1}=-c_{1}
$$

and the lemma follows.

Lemma 3. Suppose $p, q \in \mathbb{Z}$ satisfy $p<0<q$, then

$$
\tilde{D}_{h, p, q}=\frac{q}{q-p} \tilde{D}_{h, p, q-1}+\frac{-p}{q-p} \tilde{D}_{h, p+1, q}
$$

Proof. Since $p \in \mathbb{Z}$ and $p<0$, we have $p \leq-1$. In other words, for the operator $\tilde{D}_{h, p+1, q}$, we have

$$
p+1 \leq 0<q
$$

Applying Lemma 2 to $\tilde{D}_{h, p+1, q}$ gives

$$
\tilde{D}_{h, p+1, q}=D+(-1)^{q-p} \frac{h^{q-p-1}}{(q-p) !}\left(\prod_{\substack{p \leq i \leq q \\ i \neq 0}} i\right) D^{q-p}+\sum_{j=q-p+1}^{\infty} \frac{h^{j-1}}{j !} b_{j} D^{j}
$$

Similarly, for the operator $\tilde{D}_{h, p, q-1}$, we have

$$
p<0 \leq q-1
$$


and

$$
\tilde{D}_{h, p, q-1}=D+(-1)^{q-p} \frac{h^{q-p-1}}{(q-p) !}\left(\prod_{\substack{p \leq i \leq q-1 \\ i \neq 0}} i\right) D^{q-p}+\sum_{j=q-p+1}^{\infty} \frac{h^{j-1}}{j !} c_{j} D^{j} .
$$

Using (32) and (30), we have

$$
\frac{q}{q-p} \tilde{D}_{h, p, q-1}+\frac{-p}{q-p} \tilde{D}_{h, p+1, q}=D+\sum_{i=q-p+1}^{\infty} \frac{h^{i-1}}{i !} d_{i} D^{i} .
$$

Use Definition 1 and the lemma follows.

Now, we are ready for the proof of Theorem 1.

Proof [Proof of Theorem 1]. Let $q+n=m$, then the theorem becomes

$$
\tilde{D}_{h, p, p+m}=\sum_{i=0}^{n} \frac{\left(\begin{array}{c}
-p \\
i
\end{array}\right)\left(\begin{array}{c}
p+m \\
n-i
\end{array}\right)}{\left(\begin{array}{c}
m \\
n
\end{array}\right)} \tilde{D}_{h, p+i, p+m-n+i} .
$$

And the constraints become

$$
0 \leq n \leq \min (-p, p+m,-1+m) .
$$

We proceed by mathematical induction on $n$.

For the base case $n=0,(34)$ becomes

$$
\tilde{D}_{h, p, p+m}=\tilde{D}_{h, p, p+m}
$$

which is true.

For the inductive step, suppose (34) is true for $n=k$, i.e.

$$
\tilde{D}_{h, p, p+m}=\sum_{i=0}^{k} \frac{\left(\begin{array}{c}
-p \\
i
\end{array}\right)\left(\begin{array}{c}
p+m \\
k-i
\end{array}\right)}{\left(\begin{array}{c}
m \\
k
\end{array}\right)} \tilde{D}_{h, p+i, p+m-k+i} .
$$

And $k$ satisfies

$$
0 \leq k<\min (-p, p+m,-1+m) .
$$

Then $\forall i \in\{0,1, \ldots, k\}$,

$$
p+i \leq p+k<p+\min (-p, p+m,-1+m) \leq 0
$$

and

$$
p+m-k+i \geq p+m-k \geq \min (-p, p+m,-1+m)-k>0 .
$$

In other words, $\forall i \in\{0,1, \ldots, k\}$, the operator $\tilde{D}_{h, p+i, p+m-k+i}$ satisfies

$$
p+i<0<p+m-k+i \text {. }
$$


So, we can apply Lemma 3 to $\tilde{D}_{h, p+i, p+m-k+i}$ :

$$
\tilde{D}_{h, p+i, p+m-k+i}=\frac{p+m-k+i}{m-k} \tilde{D}_{h, p+i, p+m-k+i-1}+\frac{-p-i}{m-k} \tilde{D}_{h, p+i+1, p+m-k+i} .
$$

On the other hand,

$$
\frac{\left(\begin{array}{c}
-p \\
i
\end{array}\right)\left(\begin{array}{c}
p+m \\
k-i
\end{array}\right)}{\left(\begin{array}{c}
m \\
k
\end{array}\right)} \frac{p+m-k+i}{m-k}=\frac{\left(\begin{array}{c}
-p \\
i
\end{array}\right)\left(\begin{array}{c}
p+m \\
k+1-i
\end{array}\right)}{\left(\begin{array}{c}
m \\
k+1
\end{array}\right)} \frac{k+1-i}{k+1} .
$$

And

$$
\frac{\left(\begin{array}{c}
-p \\
i-1
\end{array}\right)\left(\begin{array}{c}
p+m \\
k+1-i
\end{array}\right)}{\left(\begin{array}{c}
m \\
k
\end{array}\right)} \frac{-p-i+1}{m-k}=\frac{\left(\begin{array}{c}
-p \\
i
\end{array}\right)\left(\begin{array}{c}
p+m \\
k+1-i
\end{array}\right)}{\left(\begin{array}{c}
m \\
k+1
\end{array}\right)} \frac{i}{k+1}
$$

Using (43), (44) together with (37) and (42), we have

$$
\begin{aligned}
\tilde{D}_{h, p, p+m}= & \sum_{i=0}^{k} \frac{\left(\begin{array}{c}
-p \\
i
\end{array}\right)\left(\begin{array}{c}
p+m \\
k+1-i
\end{array}\right)}{\left(\begin{array}{c}
m \\
k+1
\end{array}\right)} \frac{k+1-i}{k+1} \tilde{D}_{h, p+i, p+m-k-1+i} \\
& +\sum_{i=1}^{k+1} \frac{\left(\begin{array}{c}
-p \\
i
\end{array}\right)\left(\begin{array}{c}
p+m \\
k+1-i
\end{array}\right)}{\left(\begin{array}{c}
m \\
k+1
\end{array}\right)} \frac{i}{k+1} \tilde{D}_{h, p+i, p+m-k-1+i} \\
= & \sum_{i=0}^{k+1} \frac{\left(\begin{array}{c}
-p \\
i
\end{array}\right)\left(\begin{array}{c}
p+m \\
k+1-i
\end{array}\right)}{\left(\begin{array}{c}
m \\
k+1
\end{array}\right)} \tilde{D}_{h, p+i, p+m-k-1+i}
\end{aligned}
$$

i.e. the claim is true for $n=k+1$, which completes the inductive step.

\section{Discussion}

\subsection{Ideal weights for upwind WENO schemes}

In this subsection, we first present an example of Theorem 1. This example is taken from the fifth-order accurate WENO scheme proposed by Jiang and Shu. ${ }^{2}$ For 
Theorem 1, the linear combination corresponds to $p=-3, q=3$ and $n=2$

$$
\tilde{D}_{h,-3,2}=\sum_{i=0}^{2} \frac{\left(\begin{array}{l}
3 \\
i
\end{array}\right)\left(\begin{array}{c}
2 \\
2-i
\end{array}\right)}{\left(\begin{array}{l}
5 \\
2
\end{array}\right)} \tilde{D}_{h,-3+i, i}
$$

The ideal weights are, by Theorem 1

$$
\frac{\left(\begin{array}{l}
3 \\
0
\end{array}\right)\left(\begin{array}{l}
2 \\
2
\end{array}\right)}{\left(\begin{array}{l}
5 \\
2
\end{array}\right)}=\frac{1}{10}, \quad \frac{\left(\begin{array}{l}
3 \\
1
\end{array}\right)\left(\begin{array}{l}
2 \\
1
\end{array}\right)}{\left(\begin{array}{l}
5 \\
2
\end{array}\right)}=\frac{6}{10}, \quad \frac{\left(\begin{array}{l}
3 \\
2
\end{array}\right)\left(\begin{array}{l}
2 \\
0
\end{array}\right)}{\left(\begin{array}{l}
5 \\
2
\end{array}\right)}=\frac{3}{10} .
$$

It is clear that the result is consistent with the published work. ${ }^{2}$ Note that a fifthorder accurate TENO scheme used the same sub-stencils, ${ }^{8}$ the ideal weights in (47) are also suitable for this case.

If we consider the upwind finite difference WENO schemes, the parameters in Theorem 1 can be set to

$$
\left\{\begin{array}{l}
p=-(n+1) \\
q=n+1
\end{array}\right.
$$

And the equation becomes

$$
\tilde{D}_{h,-(n+1), n}=\sum_{i=0}^{n} \frac{\left(\begin{array}{c}
n+1 \\
i
\end{array}\right)\left(\begin{array}{c}
n \\
n-i
\end{array}\right)}{\left(\begin{array}{c}
2 n+1 \\
n
\end{array}\right)} \tilde{D}_{h,-(n+1)+i, i} .
$$

The values of the ideal weights are summarized in Table 1 from $n=1$ to $n=9$.

Table 1. Ideal weights for upwind WENO scheme $\tilde{D}_{h,-(n+1), n}$, $1 \leq n \leq 9$.

\begin{tabular}{lcc}
\hline$n$ & Order & Ideal weights \\
\hline 1 & 3 & $\frac{1}{3}, \frac{2}{3}$ \\
2 & 5 & $\frac{1}{10}, \frac{3}{5}, \frac{3}{10}$ \\
3 & 7 & $\frac{1}{35}, \frac{12}{35}, \frac{18}{35}, \frac{4}{35}$ \\
4 & 9 & $\frac{1}{126}, \frac{10}{63}, \frac{10}{21}, \frac{20}{63}, \frac{5}{126}$ \\
5 & 11 & $\frac{1}{462}, \frac{5}{77}, \frac{25}{77}, \frac{100}{231}, \frac{25}{154}, \frac{1}{77}$ \\
6 & 13 & $\frac{1}{1716}, \frac{7}{286}, \frac{105}{572}, \frac{175}{429}, \frac{175}{572}, \frac{21}{286}, \frac{7}{1716}$ \\
7 & 15 & $\frac{1}{6435}, \frac{56}{6435}, \frac{196}{2145}, \frac{392}{1287}, \frac{490}{1287}, \frac{392}{2145}, \frac{196}{6435}, \frac{8}{6435}$ \\
8 & 17 & $\frac{1}{24310}, \frac{36}{12155}, \frac{54}{12155}, \frac{2325}{12155}, \frac{882}{2431}, \frac{3528}{12155}, \frac{1176}{12155}, \frac{144}{12155}, \frac{9}{24310}$ \\
9 & 19 & $\frac{1}{92378}, \frac{45}{46189}, \frac{810}{46189}, \frac{5040}{46189}, \frac{13230}{46189}, \frac{15876}{46189}, \frac{8820}{46189}, \frac{2160}{46189}, \frac{405}{92378}, \frac{5}{46189}$ \\
\hline
\end{tabular}




\subsection{Determining ideal weights for TENO schemes}

In this subsection, we observe Theorem 1 from a different angle. The ideal weights can be regarded as partial derivatives from the perspective of expressions. More specifically, the recurrence relation, i.e. Lemma 3, can be considered as the "chain rule". This observation leads us to the fact that the ideal weights for TENO schemes can also be determined. To be more exact, the recurrence relation can be written, from the perspective of expressions, as

$$
\left\{\begin{array}{l}
\frac{\partial \tilde{D}_{h, p, q}}{\partial \tilde{D}_{h, p, q-1}}=\frac{q}{q-p} \\
\frac{\partial \tilde{D}_{h, p, q}}{\partial \tilde{D}_{h, p+1, q}}=\frac{-p}{q-p}
\end{array}\right.
$$

And Theorem 1 can be written as

$$
\frac{\partial \tilde{D}_{h, p, p+q+n}}{\partial \tilde{D}_{h, p+i, p+q+i}}=\frac{\left(\begin{array}{c}
-p \\
i
\end{array}\right)\left(\begin{array}{c}
p+q+n \\
n-i
\end{array}\right)}{\left(\begin{array}{c}
q+n \\
n
\end{array}\right)}
$$

Note that setting $n=1$ in (51) will give (50), we can use (51) to determine the ideal weights for TENO schemes. As an example, let us consider the sixth-order accurate TENO scheme. In this case, the basis schemes are

$$
\tilde{D}_{h,-3,0}, \tilde{D}_{h,-2,1}, \tilde{D}_{h,-1,2}, \tilde{D}_{h,-1,3} \text {. }
$$

We use a schematic diagram similar to one of artificial neural networks to explain the process of constructing the final scheme $\tilde{D}_{h,-3,3}$ and the method of determining the ideal weights (see Fig. 2).

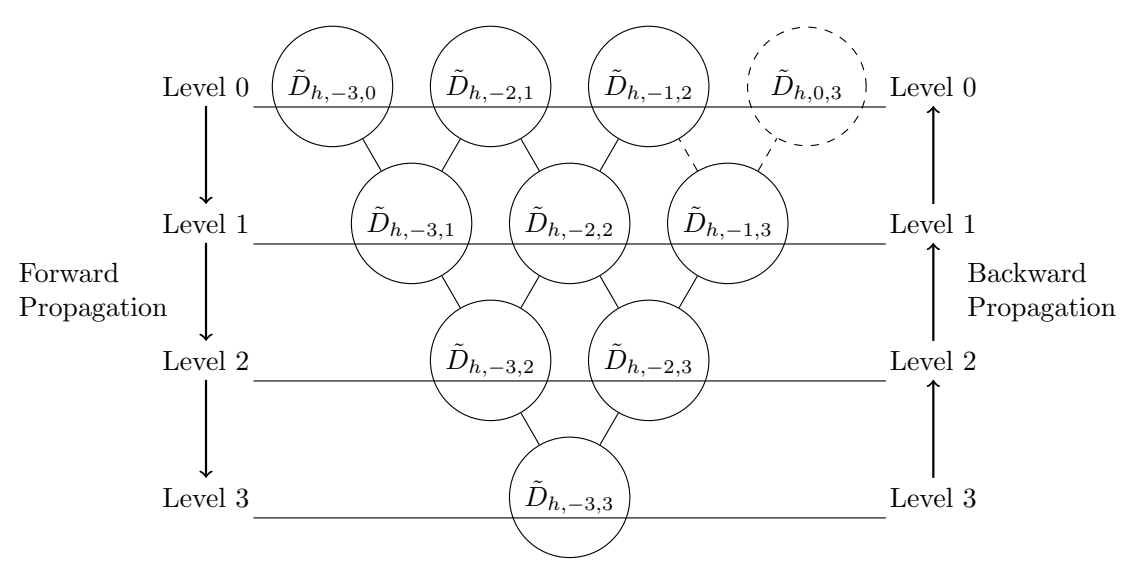

Fig. 2. Schematic diagram for the sixth-order accurate TENO scheme. 
From Fig. 2, it is clear that to construct the highest order accurate scheme (i.e. $\tilde{D}_{h,-3,3}$ ), we have to "move forward": use schemes from level 0 to construct schemes at level 1, then construct schemes at level 2 using schemes from level 1, and finally construct the only scheme at level 3 using schemes from level 2 . Note that this time a sub-stencil for the sixth-order accurate WENO scheme (i.e. the sixth-order WENO-SYMOO scheme ${ }^{11}$ ) is missing, therefore the scheme for that sub-stencil is missing (i.e. the node drawn with dashed circle, $\tilde{D}_{h, 0,3}$ ).

When we try to determine the ideal weights for the TENO basis schemes, there are two ways: move forward and move backward. Either way, Lemma 3 is actually used to determine the ideal weights. Due to the missing node, we can not always apply Theorem 1 to determine the ideal weights for TENO schemes. However, if all the lattice paths used when we perform back propagation are available, then the ideal weights given by (51) are still valid. From Fig. 2, we can deduce that for the sixth-order accurate TENO scheme, three of the ideal weights can be determined directly from (51):

$$
\left\{\begin{array}{l}
\frac{\partial \tilde{D}_{h,-3,3}}{\partial \tilde{D}_{h,-3,0}}=\frac{\left(\begin{array}{l}
3 \\
0
\end{array}\right)\left(\begin{array}{l}
3 \\
3
\end{array}\right)}{\left(\begin{array}{l}
6 \\
3
\end{array}\right)}=\frac{1}{20}, \\
\frac{\partial \tilde{D}_{h,-3,3}}{\partial \tilde{D}_{h,-2,1}}=\frac{\left(\begin{array}{l}
3 \\
1
\end{array}\right)\left(\begin{array}{l}
3 \\
2
\end{array}\right)}{\left(\begin{array}{l}
6 \\
3
\end{array}\right)}=\frac{9}{20} \\
\frac{\partial \tilde{D}_{h,-3,3}}{\partial \tilde{D}_{h,-1,3}}=\frac{\left(\begin{array}{l}
3 \\
2
\end{array}\right)\left(\begin{array}{l}
3 \\
0
\end{array}\right)}{\left(\begin{array}{l}
6 \\
2
\end{array}\right)}=\frac{1}{5} .
\end{array}\right.
$$

And the remaining ideal weight can be determined by the "chain rule", i.e.

$$
\frac{\partial \tilde{D}_{h,-3,3}}{\partial \tilde{D}_{h,-2,2}} \frac{\partial \tilde{D}_{h,-2,2}}{\partial \tilde{D}_{h,-1,2}}=\frac{\left(\begin{array}{l}
3 \\
1
\end{array}\right)\left(\begin{array}{l}
3 \\
1
\end{array}\right)}{\left(\begin{array}{l}
6 \\
2
\end{array}\right)} \frac{\left(\begin{array}{l}
2 \\
1
\end{array}\right)\left(\begin{array}{l}
2 \\
0
\end{array}\right)}{\left(\begin{array}{l}
4 \\
1
\end{array}\right)}=\frac{3}{10}
$$

We did so because one of the paths that is needed for a complete back propagation is missing, i.e.

$$
\tilde{D}_{h,-3,3} \rightarrow \tilde{D}_{h,-2,3} \rightarrow \tilde{D}_{h,-1,3} \rightarrow \tilde{D}_{h,-1,2}
$$

And this missing path is already shown in Fig. 2 (note the dashed lines and the dashed circle). In conclusion, Theorem 1 can be used to determine the ideal weights 
for TENO schemes. As a test, the ideal weights for the sixth-order accurate TENO scheme are in agreement with the published work $^{8}$ (pp. 338-339).

\section{Conclusion}

Based on the observation that two $n$th order accurate schemes can construct a $(n+1)$ th order accurate scheme by expressing the latter as a linear combination of the former, we propose a formula to determine the ideal weights which occur in finite difference WENO schemes. Although the formulation is based on nonconservative form, the formula is still valid for conservative form because a uniform mesh is assumed. Using Theorem 1, the ideal weights can be determined even if the specific expressions of the finite difference schemes on both the sub-stencils and the whole stencil are unknown. Besides, the ideal weights for TENO schemes can also be determined, if we consider the process of constructing a high order accurate scheme as a feedforward process similar to an artificial neural network. The ideal weights are, in this point of view, partial derivatives that can be determined through back propagation. Such a quantitative approach is helpful for further discussion on finite difference WENO/WENO-like schemes.

\section{Acknowledgments}

This work was supported by National Numerical Wind Tunnel project, the National Key Research and Development Program of China (2019YFA0405300) and NSFC Projects (91852203). The authors thank National Supercomputer Center in Tianjin (NSCC-TJ), and National Supercomputer Center in Guangzhou (NSCC-GZ) for providing computer time.

\section{References}

1. X.-D. Liu, S. Osher and T. Chan, J. Comput. Phys. 115, 200 (1994).

2. G. Jiang and C. Shu, J. Comput. Phys. 126, 202 (1996).

3. S. Chakravarthy, A. Harten and S. Osher, Essentially non-oscillatory shock-capturing schemes of arbitrarily-high accuracy, in 24th Aerospace Sciences Meeting (Reno, NV, USA, 1986).

4. A. Harten and S. Osher, SIAM J. Numer. Anal. 24, 279 (1987).

5. A. Harten, B. Engquist, S. Osher and S. R. Chakravarthy, J. Comput. Phys. 71, 231 (1987).

6. C.-W. Shu, SIAM Rev. 51, 82 (2009).

7. G. Gerolymos, Appl. Math. Comput. 219, 4133 (2012).

8. L. Fu, X. Y. Hu and N. A. Adams, J. Comput. Phys. 305, 333 (2016).

9. L. Fu, X. Y. Hu and N. A. Adams, J. Comput. Phys. 349, 97 (2017).

10. L. Fu, X. Y. Hu and N. A. Adams, J. Comput. Phys. 374, 724 (2018).

11. M. P. Martín, E. M. Taylor, M. Wu and V. G. Weirs, J. Comput. Phys. 220, 270 (2006). 